ARTICLE

Received 29 Nov 2015 | Accepted 3 May 2016 | Published 3 Jun 2016

DOl: $10.1038 /$ ncomms11818

OPEN

\title{
Photoelectrochemical water splitting enhanced by self-assembled metal nanopillars embedded in an oxide semiconductor photoelectrode
}

Seiji Kawasaki ${ }^{1}$, Ryota Takahashi ${ }^{1}$, Takahisa Yamamoto ${ }^{2}$, Masaki Kobayashi ${ }^{3}$, Hiroshi Kumigashira ${ }^{3}$, Jun Yoshinobu1, Fumio Komori ${ }^{1}$, Akihiko Kudo ${ }^{4,5}$ \& Mikk Lippmaa ${ }^{1}$

Production of chemical fuels by direct solar energy conversion in a photoelectrochemical cell is of great practical interest for developing a sustainable energy system. Various nanoscale designs such as nanowires, nanotubes, heterostructures and nanocomposites have been explored to increase the energy conversion efficiency of photoelectrochemical water splitting. Here we demonstrate a self-organized nanocomposite material concept for enhancing the efficiency of photocarrier separation and electrochemical energy conversion. Mechanically robust photoelectrodes are formed by embedding self-assembled metal nanopillars in a semiconductor thin film, forming tubular Schottky junctions around each pillar. The photocarrier transport efficiency is strongly enhanced in the Schottky space charge regions while the pillars provide an efficient charge extraction path. Ir-doped $\mathrm{SrTiO}_{3}$ with embedded iridium metal nanopillars shows good operational stability in a water oxidation reaction and achieves over $80 \%$ utilization of photogenerated carriers under visible light in the $400-$ to 600-nm wavelength range.

\footnotetext{
${ }^{1}$ Institute for Solid State Physics (ISSP), University of Tokyo, 5-1-5 Kashiwanoha, Kashiwa 277-8581, Japan. ${ }^{2}$ Graduate School of Engineering, Nagoya University, Furo-cho, Chikusa-ku, Nagoya 464-8603, Japan. ${ }^{3}$ Photon Factory, Institute of Materials Structure Science, High Energy Accelerator Research Organization (KEK), 1-1 Oho, Tsukuba 305-0801, Japan. ${ }^{4}$ Department of Applied Chemistry, Faculty of Science, Tokyo University of Science, 1-3 Kagurazaka, Tokyo 162-8601, Japan. ${ }^{5}$ Photocatalysis International Research Center, Research Institute for Science and Technology, Tokyo University of Science, 2641 Noda, Yamazaki 278-8510, Japan. Correspondence and requests for materials should be addressed to S.K. (email: skawasaki@sci-res.net) or to M.L. (email: mlippmaa@issp.u-tokyo.ac.jp).
} 
P otential benefits of direct hydrogen production by photoelectrochemical water splitting are well understood ${ }^{1}$, but the low energy conversion efficiency has so far prevented practical applications. The problem stems from a mismatch between the short photocarrier diffusion length and the large light absorption length in most water-stable semiconductors that are resistant to photocorrosion ${ }^{2}$. A common solution to this problem is to use various nanostructure designs that minimize the charge extraction path length and increase the photoelectrode surface area $^{3-5}$. Spontaneous nanoscale phase separation is one possible technique of nanostructure synthesis that has been used for the development of various types of functional materials, including catalysts. For example, segregation of ferromagnetic $\mathrm{CoPtCr}$ grains in a $\mathrm{CrO}_{x}$ matrix has found use in high-performance perpendicular magnetic recording media ${ }^{6}$, spontaneous formation of ferromagnetic $\mathrm{CoFe}_{2} \mathrm{O}_{4}$ nanopillars in a ferroelectric $\mathrm{BaTiO}_{3}$ matrix leads to strong strain-mediated multiferroic coupling ${ }^{7}$, and efficient solar energy conversion has been achieved in organic solar cells containing a bicontinuous network of internal donor-acceptor heterojunctions ${ }^{8}$. Spontaneous segregation of pure metals is relatively rare in oxides, but has been reported recently for $\mathrm{Fe}, \mathrm{Co}, \mathrm{Cu}$ and several noble metals ${ }^{9-12}$.

Our interest is in developing a nanocomposite material combining an $n$-type oxide semiconductor matrix with embedded three-dimensional noble metal electrodes. We show that spontaneous noble metal segregation in a perovskite oxide thin film can be used in a simple single-step deposition process to form metal-oxide nanocomposites consisting of noble metal nanopillars in a semiconducting oxide matrix. The photocarrier transport efficiency is strongly enhanced in such nanocomposites due to the formation of Schottky space charge regions around the noble metal nanopillars while the metal pillars themselves provide an efficient charge extraction path to the photocatalyst film surface. Ir-doped $\mathrm{SrTiO}_{3}$ thin films with spontaneously formed embedded Ir metal nanopillars showed good operational stability in a water oxidation reaction and achieved over $80 \%$ internal quantum efficiency under visible light illumination in the 400- to 600-nm wavelength range.

\section{Results}

Photoelectrodes with embedded metal nanopillars. We use kinetic control of epitaxial thin film growth to impose a pillarshaped structure on segregated metal inclusions, as illustrated in Fig. 1a. The metal pillars have two important roles in the photoelectrode. Since noble metals have high work functions, a Schottky barrier forms in an $n$-type oxide semiconductor in the vicinity of a metal pillar (Fig. 1c) ${ }^{3}$. The electric field in the Schottky barrier space-charge region provides the driving force for separating the photogenerated electrons from holes, reducing recombination losses. The nanopillar nanocomposite can thus efficiently separate photogenerated charges in a large volume fraction of a thin film by extending the space charge layer along the length of the nanopillar and effectively matching the charge generation depth with the optical absorption depth. The second important role of the metal nanopillars is to provide a conduction path for the generated charge to reach the liquid interface. If both the photoelectrode film and the substrate are n-type semiconductors, a Schottky barrier also forms at the interface between the metal nanopillar and the substrate, insulating the metal pillar from the substrate and driving the collected charge to the pillar-water interface. Similar design concepts have been explored before, either by embedding Pt nanopillars in an $n$-Si photoelectrode $^{13}$ or by using an inverted geometry, where semiconductor nanowires are coated with electrocatalysts
(Fig. 1b). The improvements of photoelectrochemical activity obtained with coated nanowires can be attributed to increased specific surface area and decreased charge transport distance, but the exposed nanowires are far less robust than embedded metal pillars inside a bulk semiconductor and require costly multistep fabrication procedures. The nanopillar composites explored in this work are all grown in a single deposition process, where suitable metal segregation is achieved by judicious choice of growth rate, temperature and oxygen pressure.

Growth of self-assembled epitaxial metal nanopillars. Epitaxial nanopillar composite $M(x \%): \mathrm{SrTiO}_{3}(M=\mathrm{Pt}, \mathrm{Pd}, \mathrm{Rh}, \mathrm{Au}$ and $\mathrm{Ir})$ thin films were grown by pulsed laser deposition from polycrystalline $\mathrm{SrTi}_{1-x} \mathrm{M}_{x} \mathrm{O}_{3}(x=1,3,5$ and 10\%) ablation targets. In pulsed laser deposition growth, very high supersaturation of adatoms occurs on a microsecond scale when the ablation plume hits the film surface, followed by a long relaxation period of about a second. This leads to rapid nucleation and coalescence of noble metal clusters on the substrate surface during the growth of the first unit cell of the film. If the thermodynamic (temperature and oxygen pressure) and kinetic (growth rate) parameters are suitably balanced, the metal clusters remain stable on the surface and function as gettering sites for further metal adatoms delivered to the growth front, leading to the formation of vertical metal nanopillars instead of isolated nanoparticles, as happens in the case of homogeneous bulk segregation. Our experiments show that the segregation phenomenon is surprisingly common and occurs for several metals that bond weakly to oxygen, such as noble metals, which also happen to be efficient electrocatalysts for water splitting.

Our main focus is on $\mathrm{Ir}: \mathrm{SrTiO}_{3}$ because it shows the highest photoelectrochemical activity under visible light irradiation among the metals that were tested. Intrinsic $\mathrm{SrTiO}_{3}$ with a bandgap of $3.2 \mathrm{eV}$ can only absorb ultraviolet light and is insensitive to the visible part of the spectrum. Doping with $\mathrm{Ir}^{4+}$ ions at the Ti site leads to the formation of an occupied impurity level $\sim 1.1 \mathrm{eV}$ above the $\mathrm{O} 2 p$ valence band maximum
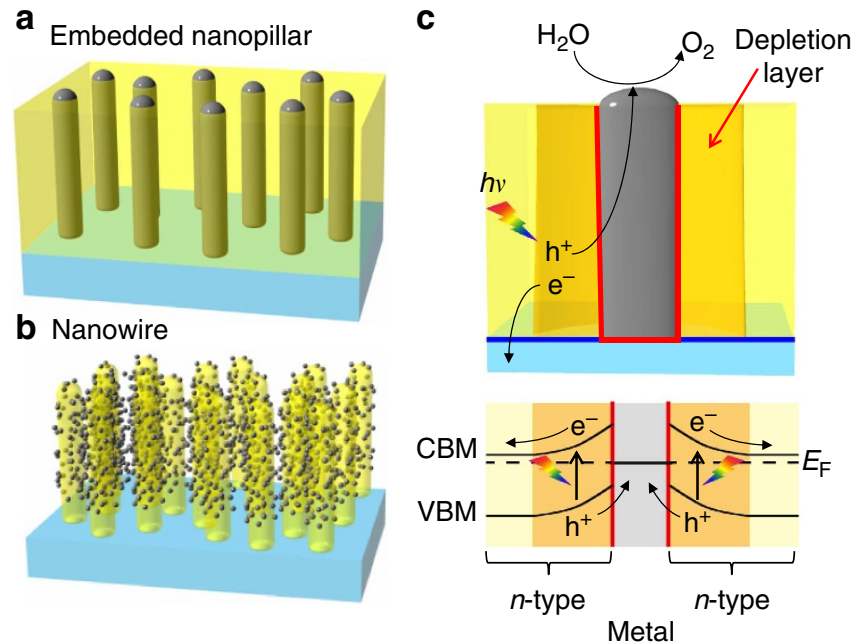

Figure 1 | Nanocomposite semiconductor photoelectrode with embedded metal nanopillars for solar water splitting. (a) Schematic illustration of embedded metal nanopillars in a semiconductor film. (b) Schematic illustration of semiconductor nanowires coated with electrocatalyst nanoparticles. (c) Schematic illustration of a tubular three-dimensional Schottky junction formed around a metal nanopillar and a qualitative band structure diagram. Red lines mark Schottky barriers and blue lines mark Ohmic interfaces. CBM, conduction band minimum; VBM, the valence band maximum energy. 


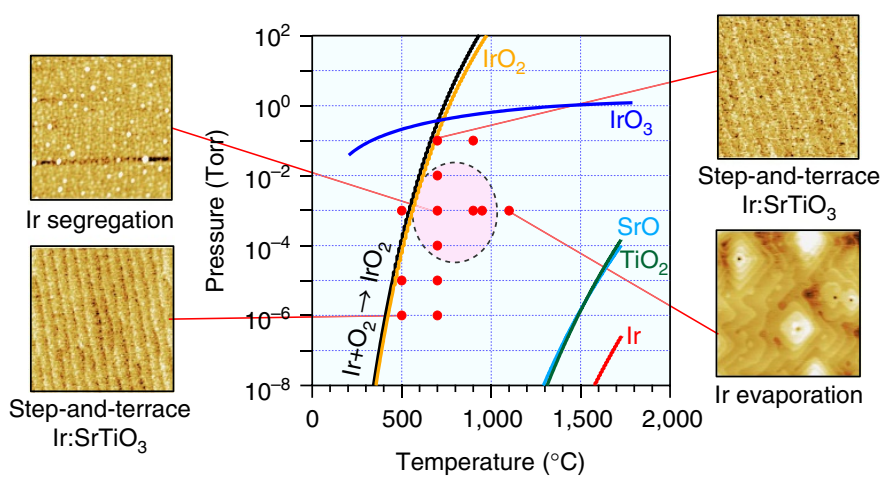

Figure 2 | The effect of temperature and oxygen pressure on the formation of Ir nanopillars in $\mathbf{S r T i O}_{3}$. Deposition conditions of Ir(5\%):SrTiO 3 films (red circles) together with vapour pressure plots of binary oxides and Ir metal ${ }^{15,16}$. The black line marks the Ir Ellingham curve ${ }^{17}$. Ir metal nanopillars formed in the region encircled with the dotted line. The AFM imaging area was $1 \times 1 \mu \mathrm{m}^{2}$. The film thickness was $20 \mathrm{~nm}$ for all samples.

and an unoccupied state $0.8 \mathrm{eV}$ below the conduction band minimum, effectively shrinking the bandgap. The Ir-doped films are dark yellow in colour and can utilize the visible part of the solar spectrum up to $\lambda \approx 700 \mathrm{~nm}, h v>1.8 \mathrm{eV}$ for photoelectrochemical reactions due to an optical transition from the occupied $\mathrm{Ir}^{4+}$ impurity level to the conduction band of $\mathrm{SrTiO}_{3}$ (ref. 14). $\operatorname{Ir}(5 \%): \mathrm{SrTiO}_{3}$ films were grown on $\mathrm{SrTiO}_{3}$ (001) substrates at various temperatures and oxygen pressures. Deposition conditions of $\operatorname{Ir}(5 \%): \mathrm{SrTiO}_{3}$ films are marked by red circles in Fig. 2, together with vapour pressure plots of binary oxides and Ir metal ${ }^{15,16}$, and the Ir Ellingham curve ${ }^{17}$. Ir metal segregation was found by atomic force microscopy (AFM) in films grown at around $700^{\circ} \mathrm{C}$ and $10^{-3}$ Torr of oxygen at an ablation pulse rate of $2 \mathrm{~Hz}$. At higher temperatures, above $1,100^{\circ} \mathrm{C}$, volatility of non-stoichiometric Ir oxides led to a loss of Ir from the films, while at lower temperatures, below $500^{\circ} \mathrm{C}$, Ir was incorporated in the $\mathrm{SrTiO}_{3}$ lattice without observable metal clustering. The growth window for the background oxygen pressure was also quite narrow. At high $\left(10^{-1}\right.$ Torr) or low $\left(10^{-6}\right.$ Torr $)$ oxygen pressures, Ir metal segregation was not observed and clear stepand-terrace film surfaces were obtained. Under the optimum conditions of $700^{\circ} \mathrm{C}$ and $10^{-3}$ Torr, an estimated $60 \%$ of Ir substituted at the Ti site of the $\mathrm{SrTiO}_{3}$ host material as $\mathrm{Ir}^{4+}$ and the remaining $40 \%$ segregated as pure Ir metal in nanopillars. The Ir metal segregation started at the initial film growth stage and was observable even in a 0.4 -nm-thick single-unit cell film (Supplementary Figs 1 and 2), which means that the nanopillars nucleated directly on the substrate surface and grew continuously through the film. The diameter of the segregated metal pillars was uniform at $\sim 10 \mathrm{~nm}$ and independent of the Ir concentration in $\mathrm{SrTiO}_{3}$. Varying the Ir-doping level between 1 and $10 \%$ changed the areal density of the pillars without having a significant effect on the average pillar diameter (Supplementary Fig. 3).

Structure analysis of nanopillar composite. X-ray diffraction revealed that the nanopillars consisted of Ir metal with the bulk face-centred cubic structure and had a cube-on-cube epitaxial relationship with the $\mathrm{SrTiO}_{3}$ host lattice (Supplementary Figs 4 and 5). X-ray photoelectron spectroscopy showed that the nanopillar films contained Ir only in the $\mathrm{Ir}^{4+}$ and metallic states (Supplementary Fig. 6). The atomic-scale structure of the Ir nanopillars was analysed by high-angle annular dark-field scanning transmission electron microscopy (HAADF-STEM). The contrast of HAADF-STEM images is proportional to the atomic weight and the bright dots observed in the plan-view and cross-section images therefore correspond to the heavier Ir metal atoms (Fig. 3). Both in-plane and out-of-plane epitaxial relationships between the pillars and the $\mathrm{SrTiO}_{3}$ matrix were
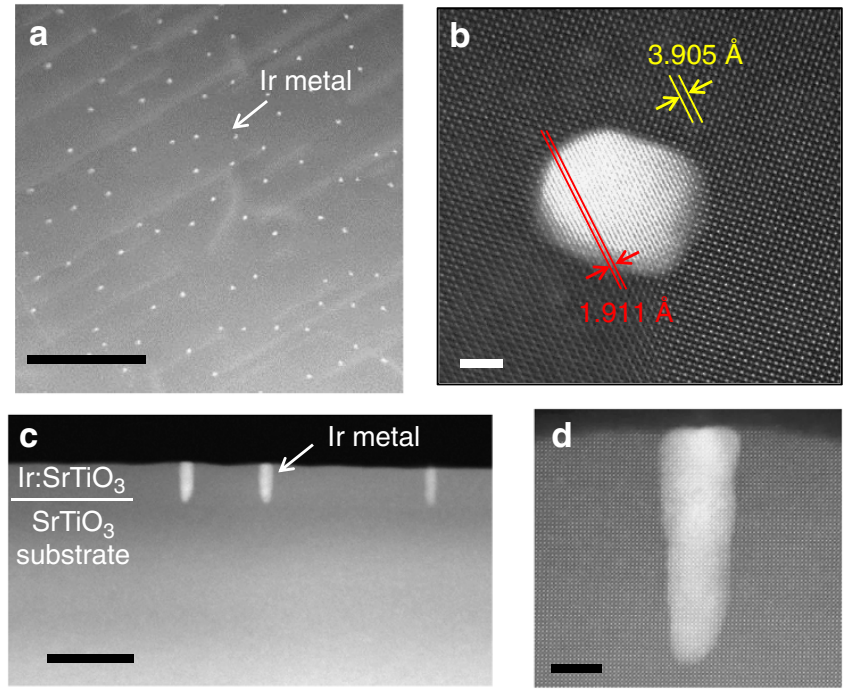

Figure 3 | Structure of epitaxial Ir nanopillars. (a) Plan-view HAADFSTEM image of an $\operatorname{Ir}(5 \%): \mathrm{SrTiO}_{3}(20 \mathrm{~nm})$ film deposited at $700^{\circ} \mathrm{C}$, $10^{-3}$ Torr. The bright dots correspond to the Ir metal pillars. Scale bar, $200 \mathrm{~nm}$. (b) A plan-view image of a single pillar shows that the Ir metal lattice is epitaxially matched with the $\mathrm{SrTiO}_{3}$ crystal lattice. The lattice parameters are $3.905 \AA$ for $\mathrm{SrTiO}_{3}$ and $3.839 \AA$ for the Ir metal. Scale bar, $2 \mathrm{~nm}$. (c,d) Wide and narrow cross-section HAADF-STEM images of the same sample. Scale bars, $50 \mathrm{~nm}$ for $\mathbf{c}$ and $5 \mathrm{~nm}$ for $\mathbf{d}$

clearly visible in the HAADF-STEM images. The STEM images show that the crystal quality of an $\mathrm{Ir}: \mathrm{SrTiO}_{3}$ film deposited at $700^{\circ} \mathrm{C}$ and $10^{-3}$ Torr was exceptionally high, with the film lattice being indistinguishable from the $\mathrm{SrTiO}_{3}$ substrate lattice, as shown in Fig. 3c,d. This is quite unexpected for films grown at a relatively low temperature, considering that even homoepitaxial $\mathrm{SrTiO}_{3}$ films grown at $700^{\circ} \mathrm{C}$ include many point defects induced by cation non-stoichiometry ${ }^{18}$. The lack of such defects in the film suggests that Ir metal segregation and rapid diffusion at the film surface suppressed defect formation in the bulk of the $\mathrm{SrTiO}_{3}$ film. The role of the Ir metal may thus be similar to a flux for $\mathrm{SrTiO}_{3}$ growth ${ }^{19}$, as indicated by the formation of $\sim 5$ unit-cellhigh $\mathrm{SrTiO}_{3}$ cones around the Ir nanopillars (Fig. 3c,d).

Schottky junctions surrounding the metal nanopillars. Since Ir metal has a very high work function of $\sim 5.7 \mathrm{eV}$ (ref. 20) and $\mathrm{SrTiO}_{3}$ is an $n$-type semiconductor with a work function of 
a

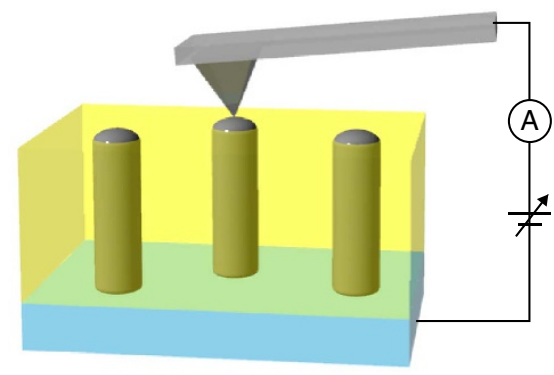

b
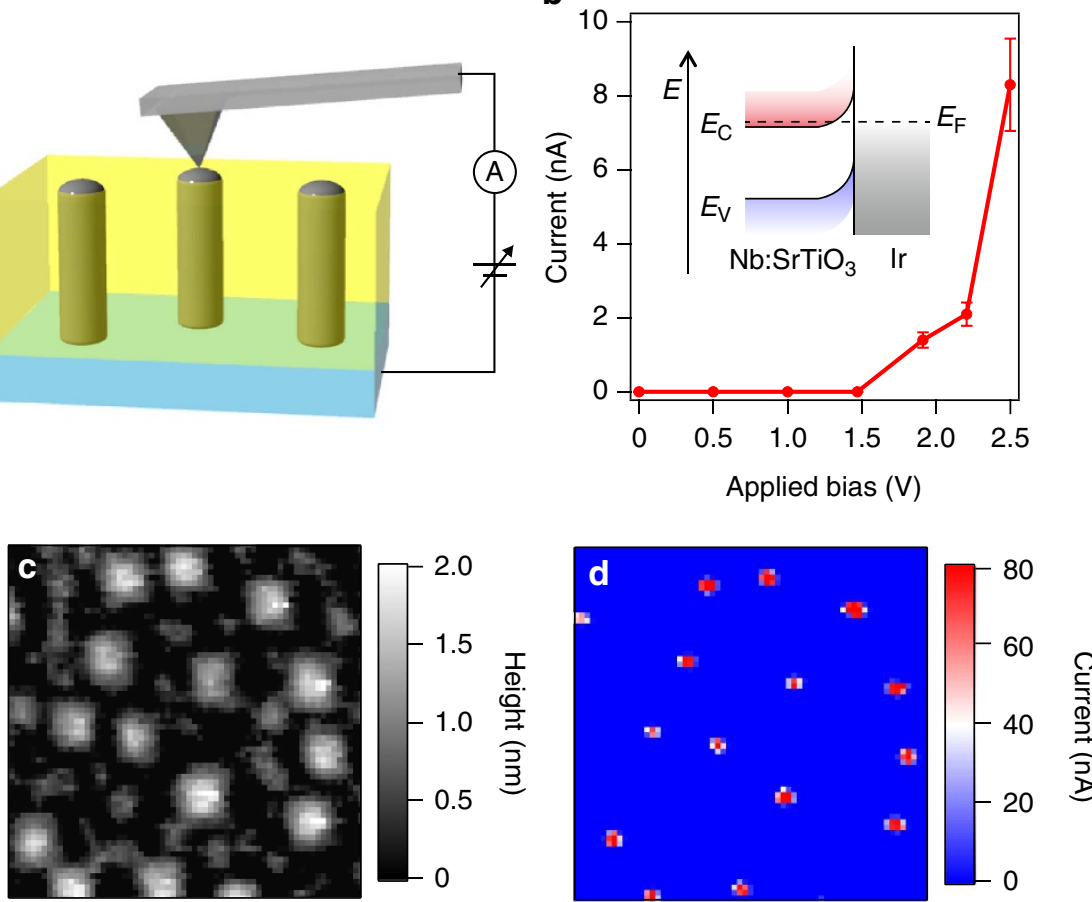

Figure 4 | Local-probe conductivity analysis of a nanopillar composite film. (a) Schematic illustration of the current mapping measurement. (b) Currentvoltage curve measured on a single Ir metal nanopillar. The inset illustrates the band diagram of the Schottky junction between an Ir pillar and the $\mathrm{Nb}(0.1 \%): \mathrm{SrTiO}_{3}$ substrate. The error bars in $\mathbf{b}$ indicate the s. d. values of the current measured at a single nanopillar site. (c) Topographic AFM image $\left(250 \times 250 \mathrm{~nm}^{2}\right)$ of an $\operatorname{tr}(5 \%): \mathrm{SrTiO}_{3}$ nanopillar film. (d) Current mapping obtained at an applied bias of $2.5 \mathrm{~V}$, simultaneously acquired with c.

4.2-4.3 eV (ref. 21), a Schottky junction forms at the pillar interface with the $\mathrm{Ir}: \mathrm{SrTiO}_{3}$ matrix. The formation of a depletion layer around the metal nanopillars increases the volume fraction of the film where strong internal electric fields enhance photocarrier separation (Fig. 1c). Local-probe transport analysis of the metal nanopillars with a conducting AFM tip (Fig. 4a) showed that the Ir nanopillars also formed a Schottky junction with the conducting $\mathrm{Nb}: \mathrm{SrTiO}_{3}$ substrate (Fig. 4b). The built-in potential was $\sim 1.5 \mathrm{~V}$, which is consistent with the work function difference between Ir metal and the $\mathrm{Nb}_{\mathrm{SrTiO}}$ substrate. Figure 4c,d shows AFM topography and simultaneously acquired current mapping images of a $\operatorname{Ir}(5 \%): \mathrm{SrTiO}_{3}$ film, measured at an applied ac bias of $2.5 \mathrm{~V}$. Metallic conductivity at the pillar positions exceeded the $\mathrm{Ir}: \mathrm{SrTiO}_{3}$ film conductivity by a factor of at least $10^{5}$. The strong upward band bending in the depletion region in the $\mathrm{Ir}: \mathrm{SrTiO}_{3}$ film surrounding the Ir nanopillars enhances the spatial separation of photogenerated electron-hole pairs and the transport of photoexcited holes from the bulk to the surface, which plays an important role in achieving an energyefficient photoelectrochemical water splitting reaction. Although the topmost surface of Ir may be oxidized, forming $\mathrm{IrO}_{2}$ at water interface, $\mathrm{IrO}_{2}$ is also an efficient electrocatalyst for water oxidation $^{22}$.

Photoelectrochemical properties of nanopillar composites. The photoelectrochemical properties of the $\operatorname{Ir}(5 \%): \mathrm{SrTiO}_{3}$ nanocomposite films were measured in a conventional three-electrode cell in a $0.1-\mathrm{M} \mathrm{KOH}$ aqueous solution $(\mathrm{pH}=13.0)$. Cyclic voltammetry curves under visible light irradiation $(\lambda>420 \mathrm{~nm})$ showed a strong anodic photocurrent (Fig. 5a), starting at $1.0 \mathrm{~V}$ versus a reversible hydrogen electrode. The sharp increase of the photocurrent is a clear indication that the photocarrier recombination rate is strongly suppressed in the bulk of the semiconductor photoelectrode. This can be attributed to the three-dimensional Schottky junctions surrounding the metal nanopillars. The optimum film thickness was $\sim 20 \mathrm{~nm}$ (Supplementary Fig. 7). The presence of the metal nanopillars was clearly detectable in Mott-Schottky plots (Supplementary Fig. 8). The photocurrent density did not decrease after 24-h operation of the photoelectrochemical cell, showing that the Ir nanopillars have good long-term operational stability (Supplementary Fig. 9). The total number of photocarriers flowing in $24 \mathrm{~h}$ $\left(2.7 \times 10^{19} \mathrm{~cm}^{-2}\right)$ is much larger than the Ir concentration of $1.7 \times 10^{15} \mathrm{~cm}^{-2}$ in a 20 -nm-thick $\operatorname{Ir}(5 \%): \mathrm{SrTiO}_{3}$ film. Therefore, the sample itself was very stable in alkaline conditions. The calculated absorbed photon-to-current efficiency and incident photon-to-current efficiency measured at $1.6 \mathrm{~V}$ versus reversible hydrogen electrode are shown in Fig. 5b together with the light absorption coefficient plot. The Ir: $\mathrm{SrTiO}_{3}$ films absorb visible light due to the Ir-induced impurity levels in the $\mathrm{SrTiO}_{3}$ bandgap $^{14}$. As expected, the incident photon-to-current efficiency curve followed the light absorption curve, showing that the photocurrent is derived from the photocarrier generation in $\mathrm{Ir}: \mathrm{SrTiO}_{3}$. The maximum absorbed photon-to-current efficiency value exceeded $80 \%$ in the 400 - to $600-\mathrm{nm}$ wavelength range.

\section{Discussion}

The present work demonstrates that self-assembled nanopillar composite photoelectrodes can be grown easily in a single-step process and provide high internal quantum efficiency in the visible spectral range for water oxidation. The embedded metal nanopillars demonstrated in this work have major advantages over other nanostructure designs: automatic formation of Schottky depletion layers in the surrounding semiconductor films, a single-step fabrication process, mechanically robust film structure, and a photocorrosion-resistant surface. These factors 

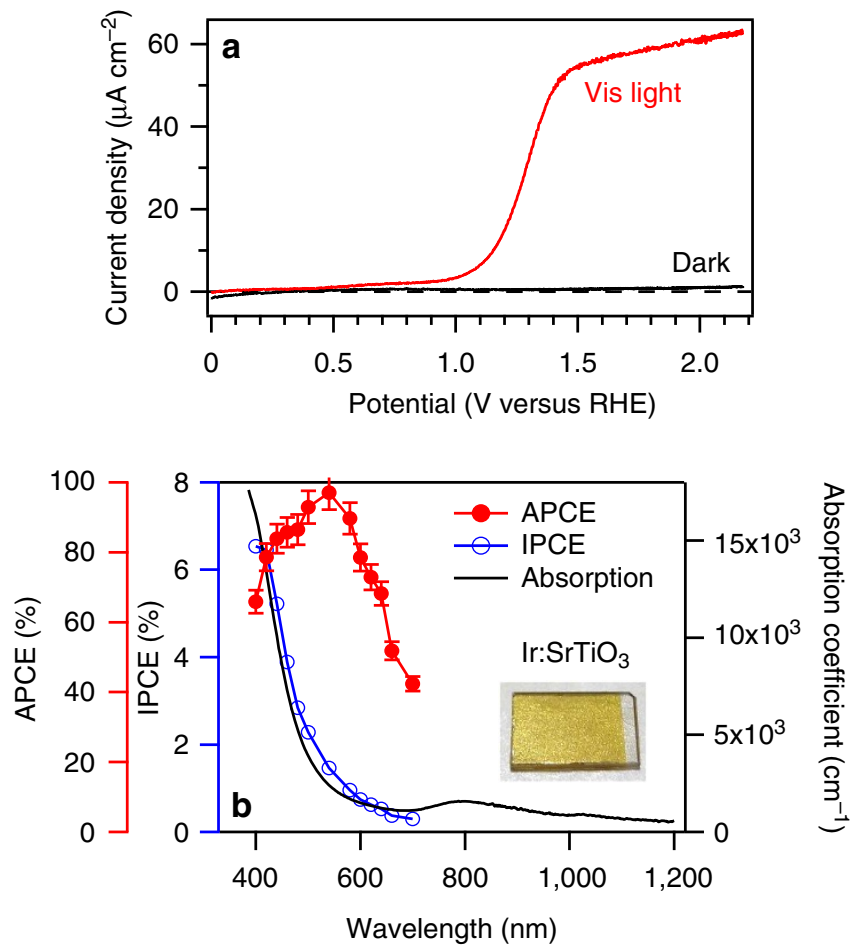

Figure 5 | Photoelectrochemical properties of an Ir:SrTiO ${ }_{3}$ film containing Ir metal nanopillars. (a) Cyclic voltammetry scans of $\operatorname{lr}(5 \%): \mathrm{SrTiO}_{3}$ nanopillar film, measured with and without light irradiation $\left(\lambda>420 \mathrm{~nm}, 70 \mathrm{~mW} \mathrm{~cm}^{-2}\right.$ ). (b) absorbed photon-to-current efficienc (APCE) and incident photon-to-current efficiency (IPCE) measured at $1.6 \mathrm{~V}$ versus reversible hydrogen electrode (RHE), together with the light absorption coefficient plot. The film thickness was $20 \mathrm{~nm}$, the estimated absorbance at $600 \mathrm{~nm}$ was $0.4 \%$. The inset shows a photograph of an Ir (5\%): $\mathrm{SrTiO}_{3}$ film deposited at $700^{\circ} \mathrm{C}, 10^{-3}$ Torr on a nondoped $\mathrm{SrTiO}_{3}(001)$ substrate. Electrolyte: $0.1 \mathrm{M} \mathrm{KOH}$ aqueous solution $(\mathrm{pH}=13.0)$; sweep rate: $20 \mathrm{mVs}^{-1}$. The error bars in $\mathbf{b}$ assume that the main source of error is the $5 \%$ uncertainty in the film thickness.

simplify the synthesis process without compromising the operational longevity of a photoelectrode. The self-assembled nanocomposites studied here may find use not only in the efficient utilization of photocarriers but also in the development of various functional devices based on nanopillars and point contacts for spintronics, memories, multiferroic composites and photonic crystals.

\section{Methods}

Sample fabrication. $\mathrm{SrTi}_{1-x} \mathrm{Ir}_{x} \mathrm{O}_{3}$ powders were synthesized in a conventional solid state reaction and pressed into pellets for use as laser ablation targets ${ }^{14}$. All films were deposited on $\mathrm{SrTiO}_{3}(001)$ or conducting $\mathrm{Nb}(0.1 \%): \mathrm{SrTiO}_{3}(001)$ substrates (Shinkosha) by pulsed laser deposition. A pulsed $\mathrm{KrF}$ excimer laser (Lambda Physik; COMPex201), operating at $248 \mathrm{~nm}, \sim 1 \mathrm{~J} \mathrm{~cm}^{-2}$ and $2 \mathrm{~Hz}$, was focused onto the target surface, creating a plasma plume that carried the evaporated material to the substrate surface. The oxygen gas pressure in the deposition chamber was adjusted with a variable leak valve from the chamber base pressure of about $5 \times 10^{-9}$ to 1 Torr. The sample temperature was monitored with an optical pyrometer operating at $2 \mu \mathrm{m}$ (Japan Sensor; FTC2) and focused onto the sample surface. The substrates were annealed at $1,000^{\circ} \mathrm{C}$ and an oxygen pressure of $1 \times 10^{-5}$ Torr for $10 \mathrm{~min}$ just before film depositions for cleaning the substrate surfaces. The film thickness was monitored by counting in situ reflection highenergy electron diffraction intensity oscillations with additional verification from the STEM images.

Scanning transmission electron microscopy. Cross-sectional and plan views were taken in an aberration-corrected (CEOS GmbH) STEM (ARM-200F, JEOL Ltd.) at $200 \mathrm{kV}$. The probe-forming aperture semiangle was $22 \mathrm{mrad}$. The HAADFSTEM images were recorded with $81-228 \mathrm{mrad}$ detectors. The cross-sectional TEM foils were prepared using a conventional method, including mechanical polishing and Ar ion milling (Model 691, Gatan Inc.). The plan-view TEM foils were prepared using a wedge-polishing method (MultiPrep system, ALLIED Inc.) and Ar ion milling.

Scanning probe microscopy. Surface topography and current mapping analysis was done with a Shimadzu SPM-9600 AFM. Topography was measured in non-contact mode with Si cantilevers (Nanoworld NCHR), while conducting PtIr $5^{-}$ coated Si cantilevers (Nanoworld NCHPt) were used for current mapping in samples grown on $\mathrm{Nb}(0.1 \%): \mathrm{SrTiO}_{3}(001)$ substrates. Measurements were done in a.c. mode with a lock-in amplifier (NF, LI5640). Variable signal amplitude at a fixed tip position was used for the $I-V$ scans.

Photoelectrochemical measurements. Film samples deposited on $\mathrm{Nb}(0.1 \%): \mathrm{SrTiO}_{3}$ (001) substrates were attached to a home-made electrochemical cell made from a polytetrafluoroethylene block (Supplementary Fig. 10), where only the film surface was exposed to the electrolyte solution $(0.1 \mathrm{M} \mathrm{KOH}$ aqueous solution, $\mathrm{pH}=13.0$ ). Dissolved oxygen was removed by $\mathrm{N}_{2}$ bubbling. Ohmic back contact to the substrate was made with an InGa alloy. The typical area of a photoelectrode surface was $0.20 \mathrm{~cm}^{2}$. The measurements were performed in a three-electrode system using Pt (Nilaco, 99.98\%) as a counter electrode and a $\mathrm{Ag} / \mathrm{AgCl}$ 3.4 M KCl (eDAQ; ET072) miniature reference electrode. Cyclic voltammetry was measured both in dark conditions and under light irradiation from a 1-kW Xe-lamp (Ushio, UXL-1000D) with an L42 cutoff filter $(\lambda>420 \mathrm{~nm})$. The photon-to-current efficiency was evaluated using monochromatized light (full-width at half-maximum of $5 \mathrm{~nm}$ ). The details are described in Supplementary Note 1. The light absorption coefficient of the $\mathrm{Ir}(5 \%): \mathrm{SrTiO}_{3}$ film was measured with an Ultraviolet/Visible/Near-infrared spectrometer (Jasco V-570) for a 350nm-thick film deposited at $700^{\circ} \mathrm{C}$ and $1 \times 10^{-3}$ Torr.

\section{References}

1. Grätzel, M. Photoelectrochemical cells. Nature 414, 338-344 (2001).

2. Manassen, J., Cahen, D. \& Hodes, G. Electrochemical, solid state, photochemical and technological aspects of photoelectrochemical energy converters. Nature 263, 97-100 (1976).

3. Liu, C., Dasgupta, N. P. \& Yang, P. Semiconductor nanowires for artificial photosynthesis. Chem. Mater. 26, 415-422 (2014).

4. Mor, G. K. et al. Enhanced photocleavage of water using titania nanotube arrays. Nano Lett. 5, 191-195 (2005).

5. Lin, Y. et al. Semiconductor nanostructure-based photoelectrochemical water splitting: a brief review. Chem. Phys. Lett. 507, 209-215 (2011).

6. Oikawa, S. et al. High performance CoPtCrO single layered perpendicular media with no recording demagnetization. IEEE Trans. Magn. 36, 2393-2395 (2000).

7. Zheng, H. et al. Multiferroic $\mathrm{BaTiO}_{3}-\mathrm{CoFe}_{2} \mathrm{O}_{4}$ nanostructures. Science 303, 661-663 (2004).

8. Yu, G., Gao, J., Hummelen, J. C., Wudl, F. \& Heeger, A. J. Polymer photovoltaic cells: enhanced efficiencies via a network of internal donor-acceptor heterojunctions. Science 270, 1789-1791 (1995).

9. Nishihata, Y. et al. Self-regeneration of a Pd-perovskite catalyst for automotive emissions control. Nature 418, 164-167 (2002).

10. Mohaddes-Ardabili, L. et al. Self-assembled single-crystal ferromagnetic iron nanowires formed by decomposition. Nat. Mater. 3, 533-538 (2004).

11. Shin, J., Goyal, A., Cantoni, C., Sinclar, J. W. \& Thompson, J. R. Self-assembled ferromagnetic cobalt/yttria-stabilized zirconia nanocomposites for ultrahigh density storage applications. Nanotechnology 23, 155602 (2012).

12. Kim, D. H. et al. A three component self-assembled epitaxial nanocomposite thin film. Adv. Func. Mater. 25, 3091-3100 (2015).

13. Aggour, M. et al. Photoactive silicon-based nanostructure by self-organized electrochemical processing semiconductor devices, materials, and processing. J. Electrochem. Soc. 154, H794-H797 (2007).

14. Kawasaki, S. et al. Electronic structure and photoelectrochemical properties of an Ir-doped $\mathrm{SrTiO}_{3}$ photocatalyst. J. Phys. Chem. C 118, 20222-20228 (2014).

15. Barin, I., Knacke, O. \& Kubaschewski, O. In Thermochemical Properties of Inorganic Substances (Springer, 1977).

16. Darling, A. S. Some properties and applications of the platinum-group metals. Int. Mater. Rev. 18, 91-122 (1973).

17. Peuckert, M. XPS study on surface and bulk palladium oxide, its thermal stability, and a comparison with other noble metal oxides. J. Phys. Chem. 89, 2481-2486 (1985).

18. Ohnishi, T. et al. Defects and transport in complex oxide thin films. J. Appl. Phys. 103, 103703 (2008).

19. Takahashi, R. et al. Perfect $\mathrm{Bi}_{4} \mathrm{Ti}_{3} \mathrm{O}_{12}$ single-crystal films via flux-mediated epitaxy. Adv. Func. Mater. 16, 485-491 (2006).

20. Michaelson, H. B. The work function of the elements and its periodicity. J. Appl. Phys. 48, 4729-4733 (1977).

21. Susaki, T., Makishima, A. \& Hosono, H. Work function engineering via $\mathrm{LaAlO}_{3} / \mathrm{SrTiO}_{3}$ polar interfaces. Phys. Rev. B 84, 115456 (2011). 
22. Chen, Y. W. et al. Atomic layer-deposited tunnel oxide stabilizes silicon photoanodes for water oxidation. Nat. Mater. 10, 539-544 (2011).

\section{Acknowledgements}

S.K. was supported by the Japan Society for the Promotion of Science Fellowship and the Program for Leading Graduate Schools (MERIT). A part of this work was supported by Nagoya University microstructural characterization platform as a programme of

"Nanotechnology Platform" of the Ministry of Education, Culture, Sports, Science and Technology (MEXT), Japan. This study was also supported by JSPS KAKENHI (Grant Nos 26105002, 25706022 and 24107004).

\section{Author contributions}

S.K. performed the sample fabrication, measurements and data analyses; S.K., R.T. and M.L. conceived and designed the project; T.Y. performed the STEM analysis; H.K. and M.K. performed the XPS analyses. All authors contributed to the discussion of the results and writing of the paper. A.K., J.Y. and F.K. jointly discussed the results.

\section{Additional information}

Supplementary Information accompanies this paper at http://www.nature.com/ naturecommunications

Competing financial interests: The authors declare no competing financial interests.

Reprints and permission information is available online at http://npg.nature.com/ reprintsandpermissions/

How to cite this article: Kawasaki, S. et al. Photoelectrochemical water splitting enhanced by self-assembled metal nanopillars embedded in an oxide semiconductor photoelectrode. Nat. Commun. 7:11818 doi: 10.1038/ncomms11818 (2016).

\section{(c) (i)}

This work is licensed under a Creative Commons Attribution 4.0 International License. The images or other third party material in this article are included in the article's Creative Commons license, unless indicated otherwise in the credit line; if the material is not included under the Creative Commons license, users will need to obtain permission from the license holder to reproduce the material. To view a copy of this license, visit http://creativecommons.org/licenses/by/4.0/ 